Joerg C. Schefold

\title{
Measurement of monocytic HLA-DR (mHLA-DR) expression in patients with severe sepsis and septic shock: assessment of immune organ failure
}

Received: 24 June 2010

Accepted: 1 July 2010

Published online: 23 July 2010

(C) Copyright jointly held by Springer and ESICM 2010

\section{J. C. Schefold (}

Department of Nephrology and Medical Intensive Care Medicine, Charité University Medicine, Charité Campus Virchow-Clinic,

Augustenburger Platz 1, 13353 Berlin, Germany

e-mail: schefold@charite.de

Tel.: +49-30-450665537

Sepsis is a under-recognised health care problem and a leading cause of death [1]. In an effort to reduce the high mortality from sepsis, large-scale clinical trials testing anti-inflammatory therapies, glucose control, low-dose corticosteroids, or activated protein $\mathrm{C}$ were performed. However, the effects of these approaches on patient mortality were rather disappointing. This may partly be due to the fact that patients were often included exclusively on the basis of a clinical diagnosis of "severe sepsis" or "septic shock" which may generate extremely heterogenous patient cohorts. When immunological interventions are tested, for example, the patient's underlying immunological state or respective dynamics should also be considered. Recently, it was therefore proposed that a reconsideration of the general concept of both sepsis research and clinical sepsis trials seems necessary and that more disease-specific trials and studies including patients on the basis of their previously defined immunological state should be performed [2-4]. Immunological biomarkers are thus needed that allow guidance of immunotherapies, risk stratification, and determination of which individuals might benefit from a given intervention.

In sepsis, the initial infection-induced pro-inflammatory host response often induces a counter-regulatory hyporesponsive immune state $[1,5]$ that resembles-by analogy with organ failures often observed in sepsis - an immune organ failure or "immunoparalysis" [6]. From a clinical perspective, it seems important to note that many patients die from a "second hit" in this later stage of sepsis. This state of sepsis-induced immunosuppression is triggered and supported by elevated levels of endotoxin and anti-inflammatory cytokines, characterised by a functional failure of innate and adaptive immunity [4], and is associated with increased mortality from sepsis [7, 8]. Data from recent immunomodulatory trials show that this condition can be reversed by both immunostimulation [9-11] or reduction of inhibitory factors [12].

Monocytes link innate and adaptive immune responses and are key immune cells in sepsis. Major findings in sepsis-induced immunosuppression include reduced monocytic phagocytotic activity, changes in monocytic cytokine expression profiles towards an anti-inflammatory phenotype (e.g. increased IL-10 expression), diminished monocytic antigen presentation via the major histocompatibility (MHC) class II complex [4], lymphocytic dysfunction and apoptosis-induced loss of circulating Tand B-lymphocytes [13]. In particular, a diminished surface expression of human leukocyte antigen (HLA)-DR molecules on monocytes (mHLA-DR) was proposed to serve as a sensitive (functional) surrogate index for sepsis-induced immunosuppression (reviewed in [4]). Rather than single inflammatory mediators which often are pleiotropic and redundant, functional immunity as assessed via mHLA-DR may reflect the net sum of pro- and anti-inflammatory influences and thus the actual immunological phenotype/phase of sepsis. Importantly, as functional immunity is a dynamic process, the expression of mHLA-DR must not be understood as a static index.

The concept that a reduced mHLA-DR expression reflects sepsis-induced immunosuppression seems generally well accepted and an association of mHLA-DR with clinical outcome (i.e. mortality or measures of morbidity) was observed in most of the larger analyses. Nevertheless, 
not all authors found such associations (discussed in [4, $14,15])$. These differences might be due to a number of reasons and may at least partially arise from different points in time of sample drawing (early vs. late). As sepsis-induced immunosuppression is a phenomenon of "late sepsis", the predictive power of mHLA-DR should be investigated in samples taken in the later, rather than in the very early course of sepsis (i.e. less than $48 \mathrm{~h}$ after establishing of the diagnosis). Alternatively, the recovery slope of a diminished mHLA-DR expression seems interesting to investigate [16]. Second, specific (pre)analytical requirements for flow cytometric mHLA-DR analysis apply [17, 18]. Both prolonged sample transportation times (more than $4 \mathrm{~h}$ ) and transportation at room temperature induce a non-specific mHLA-DR increase and may impede precise measurements. Third, flow cytometric mHLA-DR assessment should be performed by using a newly available standardised quantitative test (mHLA-DR in molecules/cell; coefficient of variation: intra-lab 3\%, inter-lab 18\%) [17]. This may allow interlab data comparison. Fourth, as mentioned before, mHLA-DR modulation during sepsis is dynamic and consecutive measurements are required for reliable determination of baseline levels in septic individuals [7].

In this issue of Intensive Care Medicine, Landelle et al. put the clinical relevance of mHLA-DR measurements into a new perspective [19]. In a prospective single-centre observational trial, the authors investigated whether a diminished mHLA-DR expression is associated with an increased incidence of nosocomial infections (NI) in 209 patients with septic shock. After adjustment for clinical confounders, it was found that persistent low mHLA-DR expression is independently associated with NI development. A significant lower mHLA-DR expression (expressed as mean flourescence intensities, MFI) was found in non-surviving patients $(n=51)$ when compared to survivors $(n=102)$ from septic shock (33 vs. 67, $p<0.001)$. Flow cytometric assessment of mHL-DR was performed at days 3-4 $(n=153)$ and 6-9 after the onset of septic shock. At day 3-4 after the onset of shock, patients who developed NI $(n=37)$ had significantly lower mHLA-DR values (39 vs. 65 , given as MFI, $p=0.008)$ than those patients who did not acquire NI $(n=116)$. Receiver operating curve analyses revealed that an mHLA-DR expression less than 54 (given as MFI) was the best cut-off to predict NI development (area under curve 0.65 , sensitivity $68 \%$, specificity $62 \%$ ). A adjusted hazards ratio (aHR) of 2.52 was calculated $(95 \%$ confidence interval 1.20-5.30, $p=0.02$ ). At day 6-9, low mHLA-DR (less than 57, expressed as MFI) remained independently associated with NI development (aHR $2.18,95 \%$ confidence interval $1.04-4.59, p=0.04)$. A reduced mHLA-DR expression may thus predict the risk for NI development at both days 3-4 and 6-9 after the onset of shock.

This largest study on the issue supports the initial clinical observation that patients with sepsis-induced immunosuppression are indeed at a higher risk to develop secondary infections. Nevertheless, a number of questions remain unanswered that stimulate further research. First, the optimal timing for the prediction of NI remains unclear. Second, the data on mHLA-DR expression are given in mean fluorescence intensities/percentage of positive cells as they were obtained in the era prior to the development of the standardized quantitative assay. Transposing of the data/mHLA-DR thresholds may thus be difficult and the results should be reproduced in future analyses using the standardized mHLA-DR assay. Last, patients were included at the start of vasopressor treatment which, from a clinical perspective, may not accurately reflect the onset of sepsis/shock. Nevertheless, this remains a major problem in sepsis research as the exact point in time of onset of sepsis can often only be estimated.

Importantly, Landelle et al. [19] add to the mounting data from numerous groups that severe immunological impairments often occur in sepsis. These alterations are of significant clinical relevance and constitute a new organ failure that, like other organ failures, deserves assessment and most likely treatment in the future.

Conflict if interest statement The author declares that he has no competing interests.

\section{References}

1. Annane D, Bellissant E, Cavaillon JM (2005) Septic shock. Lancet 365:63-78

2. Carlet J, Cohen J, Calandra T, Opal SM, Masur H (2008) Sepsis: time to reconsider the concept. Crit Care Med 36:964-966

3. Schefold JC, Hasper D, Reinke P, Monneret G, Volk HD (2008) Consider delayed immunosuppression into the concept of sepsis. Crit Care Med $36: 3118$
4. Monneret G, Venet F, Pachot A, Lepape A (2008) Monitoring immune dysfunctions in the septic patient: a new skin for the old ceremony. Mol Med 14:64-78

5. Cohen J (2002) The immunopathogenesis of sepsis. Nature 420:885-891

6. Volk HD, Reinke P, Docke WD (2000) Clinical aspects: from systemic inflammation to 'immunoparalysis'. Chem Immunol 74:162-177
7. Monneret G, Lepape A, Voirin N, Bohe J, Venet F, Debard AL, Thizy H, Bienvenu J, Gueyffier F, Vanhems P (2006) Persisting low monocyte human leukocyte antigen-DR expression predicts mortality in septic shock. Intensive Care Med 32:1175-1183 
8. Caille V, Chiche JD, Nciri N, Berton C, 12. Schefold JC, von Haehling S, Corsepius Gibot S, Boval B, Payen D, Mira JP, Mebazaa A (2004) Histocompatibility leukocyte antigen-D related expression is specifically altered and predicts mortality in septic shock but not in other causes of shock. Shock 22:521-526

9. Docke WD, Randow F, Syrbe U, Krausch D, Asadullah K, Reinke P, Volk HD, Kox W (1997) Monocyte deactivation in septic patients: restoration by IFN-gamma treatment. Nat Med 3:678-681

10. Nierhaus A, Montag B, Timmler N, Frings DP, Gutensohn K, Jung R, Schneider CG, Pothmann W, Brassel AK, Schulte Am Esch J (2003) Reversal of immunoparalysis by recombinant human granulocyte-macrophage colony-stimulating factor in patients with severe sepsis. Intensive Care Med 29:646-651

11. Meisel C, Schefold JC, Pschowski R, Baumann T, Hetzger K, Gregor J, Weber-Carstens S, Hasper D, Keh D, Zuckermann H, Reinke P, Volk HD (2009) Granulocyte-macrophage colony-stimulating factor to reverse sepsis-associated immunosuppression: a double-blind, randomized, placebocontrolled multicenter trial. Am J Respir Crit Care Med 180:640-648
M, Pohle C, Kruschke P, Zuckermann H, Volk HD, Reinke P (2007) A novel selective extracorporeal intervention in sepsis: immunoadsorption of endotoxin, interleukin 6, and complementactivating product $5 \mathrm{a}$. Shock 28:418-425

13. Hotchkiss RS, Tinsley KW, Swanson PE, Schmieg RE Jr, Hui JJ, Chang KC, Osborne DF, Freeman BD, Cobb JP, Buchman TG, Karl IE (2001) Sepsisinduced apoptosis causes progressive profound depletion of $\mathrm{B}$ and $\mathrm{CD} 4+\mathrm{T}$ lymphocytes in humans. J Immunol 166:6952-6963

14. Perry SE, Mostafa SM, Wenstone R, Shenkin A, McLaughlin PJ (2003) Is low monocyte HLA-DR expression helpful to predict outcome in severe sepsis? Intensive Care Med 29:1245-1252

15. Schefold JC, Hasper D, Volk HD, Reinke P (2008) Sepsis: time has come to focus on the later stages. Med Hypotheses 71:203-208

16. Lukaszewicz AC, Grienay M, RescheRigon M, Pirracchio R, Faivre V, Boval B, Payen D (2009) Monocytic HLA-DR expression in intensive care patients: interest for prognosis and secondary infection prediction. Crit Care Med 37:2746-2752
17. Docke WD, Hoflich C, Davis KA, Rottgers K, Meisel C, Kiefer P, Weber SU, Hedwig-Geissing M, Kreuzfelder E, Tschentscher P, Nebe T, Engel A, Monneret G, Spittler A, Schmolke K, Reinke P, Volk HD, Kunz D (2005) Monitoring temporary immunodepression by flow cytometric measurement of monocytic HLA-DR expression: a multicenter standardized study. Clin Chem 51:2341-2347

18. Monneret G, Elmenkouri N, Bohe J, Debard AL, Gutowski MC, Bienvenu J, Lepape A (2002) Analytical requirements for measuring monocytic human lymphocyte antigen DR by flow cytometry: application to the monitoring of patients with septic shock. Clin Chem 48:1589-1592

19. Landelle C, Lepape A, Voirin N, Tognet E, Venet F, Bohe J, Vanhems P, Monneret G (2010) Low monocyte human leukocyte antigen-DR is independently associated with nosocomial infections after septic shock. Intensive Care Med doi: 10.1007/s00134-010-1962-х 